\title{
Ampelopsis japonica Makino Extract Inhibits the Inflammatory Reaction Induced by Pathogen-Associated Molecular Patterns in Epidermal Keratinocytes
}

\author{
Mi-Ra Choi ${ }^{1}$, Dae-Kyoung Choi ${ }^{1}$, Ki-Duck Kim ${ }^{1}$, Sue Jeong Kim ${ }^{1}$, Dong-Il Kim², Myung Im $^{1}$, \\ Young Lee ${ }^{1}$, Young-Joon Seo ${ }^{1}$, Chang Deok Kim ${ }^{1}$, Jeung-Hoon Lee ${ }^{1,3}$ \\ ${ }^{1}$ Department of Dermatology, Chungnam National University School of Medicine, Daejeon, ${ }^{2}$ Department of Obstetrics and Gynecology, \\ Dongguk University College of Korean Medicine, Seoul, ${ }^{3}$ Skin Med Company, Daejeon, Korea
}

Background: Keratinocytes are the major cells in epidermis, providing barrier components such as cornified cells through the sophisticated differentiation process. In addition, keratinocytes exerts their role as the defense cells via activation of innate immunity. It has been known that pathogen-associated molecular patterns (PAMPs) including double-strand RNA and nucleotides can provoke inflammatory reaction in keratinocytes. Objective: The aim of this study is to evaluate the effect of Ampelopsis japonica Makino extract (AE) on PAMPs-induced inflammatory reaction of keratinocytes. Methods: The effects of $\mathrm{AE}$ were determined using poly (I:C)-induced inflammation and imiquimod-induced psoriasiform dermatitis models. Results: In cultured keratinocytes, AE significantly inhibited poly(I:C)-induced expression of inflammatory cytokines, such as interleukin (IL)- $1 \beta$, IL-6, IL-8 and tumor necrosis factor- $\alpha$. AE significantly inhibited poly(l:C)-induced release of caspase-1 active form (p20), and down-regulated nuclear factor- $\kappa \mathrm{B}$ signaling pathway. In imiquimod-induced psoriasiform dermatitis model, topical application of $A E$ resulted in significant reduction of epidermal hyperplasia. Conclusion: These results suggest that $\mathrm{AE}$ may

Received May 15, 2015, Revised August 14, 2015, Accepted for publication August 17, 2015

Corresponding author: Jeung-Hoon Lee, Department of Dermatology, Chungnam National University School of Medicine, 266 Munhwa-ro, Jung-gu, Daejeon 35015, Korea. Tel: 82-42-280-7707, Fax: 82-42255-5098, E-mail: jhoon@cnu.ac.kr

This is an Open Access article distributed under the terms of the Creative Commons Attribution Non-Commercial License (http://creativecommons. org/licenses/by-nc/4.0) which permits unrestricted non-commercial use, distribution, and reproduction in any medium, provided the original work is properly cited.

Copyright (c) The Korean Dermatological Association and The Korean Society for Investigative Dermatology be a potential candidate for the treatment of skin inflammation. (Ann Dermatol 28(3) 352 359, 2016)

\section{-Keywords-}

Ampelopsis, Inflammation, Keratinocytes, Pathogen-associated molecular pattern molecules

\section{INTRODUCTION}

Skin lies between organism and environment. It provides protective barrier that prevents the entrance of external pathogens from outside as well as the water loss from inside $^{1}$. This protective function is primarily supported by the cornified cells, which are formed by the sophisticated process of keratinocyte differentiation. During the differentiation process, the keratinocyte progenitor cells in the basal layer of epidermis proliferate and move upwardly, and the differentiation process begins in the suprabasal layers. Extensive but highly coordinated gene expression change takes place in a spatio-temporal manner. For example, many differentiation-related genes such as involucrin, loricrin and filaggrin are expressed with the pattern of increase in later stage of keratinocyte differentiation $^{2-4}$.

In addition to their essential role for physical barrier, epidermal keratinocytes play an important role as the primary defense cells in innate immunity. For example, keratinocytes express a variety of pattern recognition receptors (PRRs) such as Toll-like receptors (TLRs). These PRRs can recognize the bacterial pathogen-associated molecular patterns (PAMPs) as well as the damage-associated molecular patterns (DAMPs), leading to the activation of in- 
flammation-related intracellular signaling and production of inflammatory cytokines from keratinocytes ${ }^{5-7}$.

Psoriasis is a common skin disease that occurs at an incidence of $0.5 \% \sim 3 \%$ of the population worldwide. In psoriatic skin, hyperproliferation of basal layer keratinocytes as well as suprabasal layer keratinocytes is observed, and keratinocyte differentiation is largely disturbed. In addition, inflammatory reaction is highly increased in the affected skin area $^{8}$. Since that immunosuppressant cyclosporine A efficiently blocks the activation of $\mathrm{T}$ cells and consequently improves the psoriatic symptoms, it is generally believed that psoriasis is a Th1-type immune cell-mediated disease ${ }^{9}$. In addition, recent findings underscore the important role of keratinocytes in the pathophysiology of psoriasis. For instance, psoriasis can be triggered and/or exacerbated by physical trauma on the skin, suggesting that keratinocytes may be the potential origin cells for psoriasis. Stimulation of keratinocytes with various PAMPs or DAMPs results in activation of innate immunity, leading to production of inflammatory cytokines related with psoriasis ${ }^{10-12}$. In this regard, the inflammatory reaction of keratinocytes can be a good target for the development of novel therapeutics on psoriasis.

We attempted to find therapeutics on psoriasis using PAMPs-induced inflammation model, and found that Ampelopsis japonica Makino extract (AE) has an inhibitory potential on inflammatory reaction. The $A E$ has been shown to have anti-metastatic effect in human breast cancer cell line ${ }^{13}$. However, the effect of AE on inflammatory reaction in epidermal keratinocytes remains to be elucidated. In this study, we demonstrate that AE inhibits PAMPs-induced inflammatory reaction in keratinocytes, suggesting that $\mathrm{AE}$ can be applicable for psoriasis treatment.

\section{MATERIALS AND METHODS}

\section{Cell culture}

Simian virus 40 large $T$ antigen-transformed human epidermal keratinocytes (SV-HEKs) were cultured in keratinocyte-serum free medium supplemented with bovine pituitary extract (BPE) and recombinant human epidermal growth factor (rhEGF) (Life Technologies Corporation, Grand Island, NY, USA). For treatment with poly(l:C), keratinocytes were incubated with MCDB153 medium (Biochrom GmbH, Berlin, Germany) supplemented with $\mathrm{BPE}$ and rhEGF for overnight, then poly(I:C) (InvivoGene, San Diego, CA, USA) was added to the medium at the final concentration of $1 \mu \mathrm{g} / \mathrm{ml}$.

\section{Preparation of Ampelopsis japonica Makino extract}

The air-dried root of Ampelopsis japonica Makino (Omniherb Co., Daegu, Korea) was cut into pieces and extracted with 8 volumes of boiling-water for 4 hours. The extract was filtered using Whatman paper, evaporated under reduced pressure condition, and then freeze-dried (Eyela, Irvine, CA, USA). For treatment of cultured cells, $\mathrm{AE}$ was reconstituted in distilled water and filter-sterilized. For topical application in animal model, AE was reconstituted in polyethylene glycol (PEG).

\section{Cytotoxicity test}

SV-HEKs were seeded in 6-well plate at a density of $2 \times 10^{5}$, treated with AE for 24 hours. After treatment, cells received $2 \mathrm{mg} / \mathrm{ml}$ MTT (3-(4,5-dimethylthiazol-2-yl)-2,5-diphenyltetrazolium bromide) solution and were incubated for a further 4 hours. The medium was removed and the resulting formazan crystal was solubilized in $100 \mu \mathrm{l}$ of dimethyl sulfoxide. Cell viability was determined by measuring optical density at $540 \mathrm{~nm}$ using an enzyme-linked immunosorbent assay (ELISA) reader. For lactate dehydrogenase (LDH) activity measurement, culture medium were collected and enzyme activity was determined using Cytotoxicity detection kit (Roche, Mannheim, Germany).

\section{Quantitative real-time polymerase chain reaction}

Total RNAs were isolated using easy-Blue RNA extraction kit (Intron, Daejeon, Korea). Two $\mu \mathrm{g}$ of total RNAs were reverse transcribed with moloney-murine leukaemia virus reverse transcriptase (Elpis Biotech, Daejeon, Korea). Aliquots of RT mixture were amplified using SYBR Green real-time polymerase chain reaction master mix (Elpis Biotech). The following primers sequences were used: interleukin (IL)-1 $\beta$, 5'-TTAAAGCCCGCCTGACAGA and 5'GCGAATGACAGAGGGTTTCTTAG; IL-6, 5'-CTGCGCAGCTTTAAGGAGTTC and 5'-CCATGCTACATTTGCCGAAGA; IL-8, 5'-CСТTTCCACCCCAAATTTATCA and 5'-TTTCTGTGTTGGCGCAGTGT; tumor necrosis factor (TNF)- $\alpha$, 5'CTCCTTCAGACACCCTCAACCT and 5'-CGACCCTAAGCCCCCAATT; glyceraldehyde-3-phosphate dehydrogenase, 5'-TGCACCACCAACTGCTTAGC and 5'-GGCATGGACTGTGGTCATGAG.

\section{ELISA}

Culture medium was collected, and secreted IL-1 $\beta$ and TNF- $\alpha$ were determined using commercial ELISA kits. ELISA kits were purchased from R\&D Systems (Minneapolis, $M N$, USA). 


\section{Western blotting}

Cells were lysed in Proprep solution (Intron). Total protein was measured using a bicinchoninic acid protein assay kit (Pierce Biotechnology, Rockford, IL, USA). Samples were run on sodium dodecyl sulfate-polyacrylamide gels, transferred to nitrocellulose membranes and incubated with appropriate antibodies. Blots were then incubated with peroxidase-conjugated secondary antibodies, visualized by enhanced chemiluminescence (Intron). For determination of secreted proteins, cell culture medium was concentrated using a Protein concentration kit (Elpis Biotech). The following primary antibodies were used in this study: phospho-p65, caspase-1 (Cell Signaling Technology, Beverly, MA, USA); actin (Sigma-Aldrich, St. Louis, MO, USA).

\section{Animal test}

Male BALB/C mice at $6 \sim 8$ weeks of age were purchased from Orient Bio (Seongnam, Korea). The psoriasiform skin inflammation was generated by topical application of $5 \%$ imiquimod cream (Aldara; 3M Health Care Ltd., Leicestershire, United Kingdom) daily for 7 days ${ }^{14}$. AE was dissolved in PEG to the desired concentration, and pretreated 1 hour before imiquimod application.

\section{Immunohistochemistry}

Paraffin sections were incubated with $\mathrm{H}_{2} \mathrm{O}_{2}$ for 10 minutes to block endogenous peroxidase and then soaked in $5 \%$ bovine serum albumin for 30 minutes. Sections were incubated with anti-IL-1 $\beta$ antibody (Abcam, Cambridge, $\mathrm{MA}$ ) and anti-proliferating cell nuclear antigen (PCNA) an-

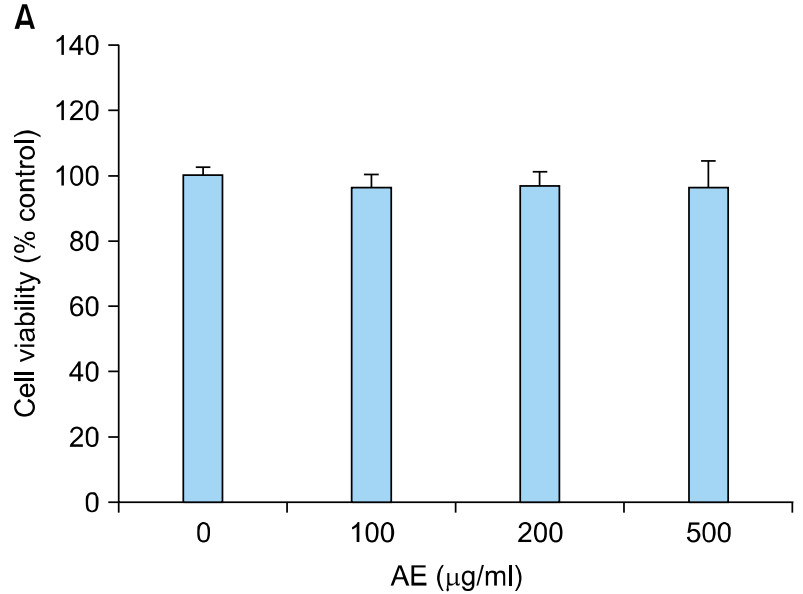

tibody (Abcam). Sections were incubated sequentially with peroxidase-conjugated secondary antibody and visualized with Chemmate envision detection kit (Dako, Carpinteria, CA, USA).

\section{Statistical analysis}

Data were evaluated statistically using one-way analysis of variance (ANOVA) with the IBM SPSS Statistics ver. 22.0 (IBM Co., Armonk, NY, USA). Statistical significance was set at $p<0.01$.

\section{RESULTS}

In an attempt to find potential therapeutics for psoriasis, we found that $A E$ has an inhibitory potential on inflammatory reaction of keratinocytes. We first determined the cytotoxicity of $\mathrm{AE}$ on keratinocytes cultured in vitro. MTT assay showed that AE did not induce cell death up to the dose of $500 \mu \mathrm{g} / \mathrm{ml}$ (Fig. 1A). In LDH assay, higher concentration of $\mathrm{AE}(500 \mu \mathrm{g} / \mathrm{ml})$ resulted in slight increase of $\mathrm{LDH}$ release, indicating the damage of the plasma membrane (Fig. 1B). Thus, we treated keratinocytes up to the concentration of $200 \mu \mathrm{g} / \mathrm{ml}$ of $\mathrm{AE}$ in consecutive experiments.

Double-strand RNAs are generally provided external viruses, and they act as the PAMP in innate immune response. It has been suggested that psoriatic keratinocytes show increased sensitivity to viral RNA intermediates, thereby leading to excessive proinflammatory response and maintenance of the inflammatory skin phenotype $^{11}$. Poly $(\mathrm{l}: \mathrm{C})$ is a synthetic analogue of double-stranded RNA that is used widely to mimic viral

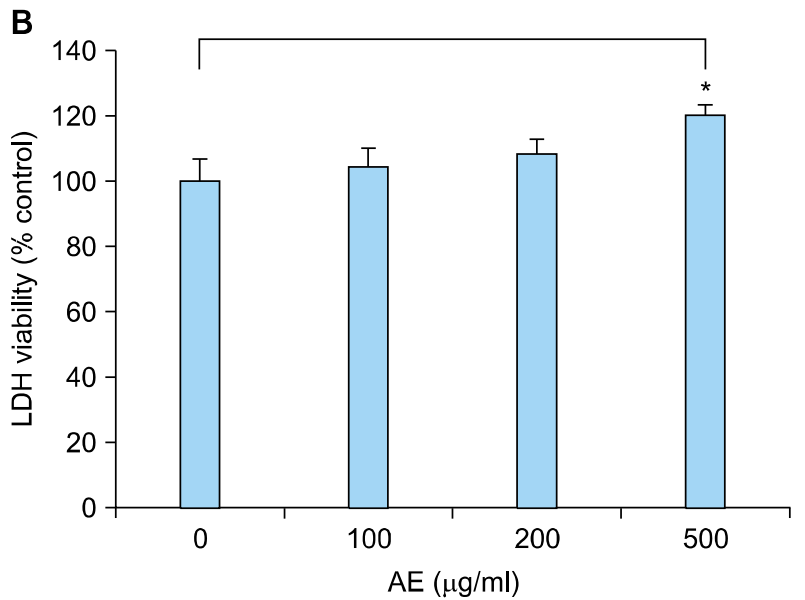

Fig. 1. Cytotoxicity of Ampelopsis japonica Makino extract (AE). (A) SV40Tag-transformed human epidermal keratinocytes (were treated with $\mathrm{AE}$ at the indicated concentrations for 24 hours. Cell viability was measured by MTT assay. (B) Lactate dehydrogenase (LDH) assay. After treatment with $\mathrm{AE}$, culture medium was collected and $\mathrm{LDH}$ activity was determined. The mean values \pm standard deviations are averages of triplicate measurements. ${ }^{*} p<0.01$. 
infection. Poly $(\mathrm{I}: \mathrm{C})$ induces innate immune response in a TLR3-dependent manner ${ }^{11,15}$. We tested the effect of $A E$ on poly(l:C)-induced immune response of keratinocytes. Poly (I:C) increased expression of inflammation-related cytokines including IL-1 $\beta$, IL-6, IL-8 and TNF- $\alpha$ from keratinocytes. Pretreatment of $\mathrm{AE}$ significantly inhibited the poly(I:C)-induced cytokine expression (Fig. 2). Consistent with these results, poly $(\mathrm{I}: \mathrm{C})$ increased secretion of IL-1 $\beta$ and TNF- $\alpha$ from keratinocytes, and AE pretreatment significantly inhibited poly(l:C)-induced cytokine release (Fig. 3).

It has been established that activation of TLR3 induces IL-1 $\beta$ production via an inflammasome-dependent mechanism in keratinocytes ${ }^{15}$. Poly(I:C) induced IL-1 $\beta$ secretion from keratinocytes, together with inflammasome-activated caspase-1 (p20). To investigate the action mechanism of $A E$, we examined the inflammasome activation by determining the release of active form of caspase-1 (p20).
As expected, poly $(\mathrm{I}: \mathrm{C})$ induced the secretion of caspase-1 (p20), and pretreatment with AE significantly inhibited the secretion of caspase-1 (p20) (Fig. 4A). We also checked the effect of $A E$ on nuclear factor (NF)- $\kappa B$ signaling, the central player in inflammatory reaction. Poly $(\mathrm{l}: \mathrm{C})$ increased NF- $\kappa$ B activity in terms of phosphorylation of p65 subunit. AE pretreatment significantly inhibited poly(l:C)-induced phosphorylation of p65 in a dose-dependent manner (Fig. 4B).

To further evaluate the potential effect of $A E$, we performed animal test. We used a well-established imiquimod-induced psoriasiform dermatitis model ${ }^{16}$. Imiquimod is an analogue of adenosine, which exerts its action as the PAMP specific for TLR7 ${ }^{17}$. Daily topical application of $5 \%$ imiquimod cream (Aldara) induced psoriasiform dermatitis, in which epidermal thickness was remarkably increased. When $\mathrm{AE}$ was pretreated, induction of psoriasiform dermatitis was significantly inhibited. Histochemistry study
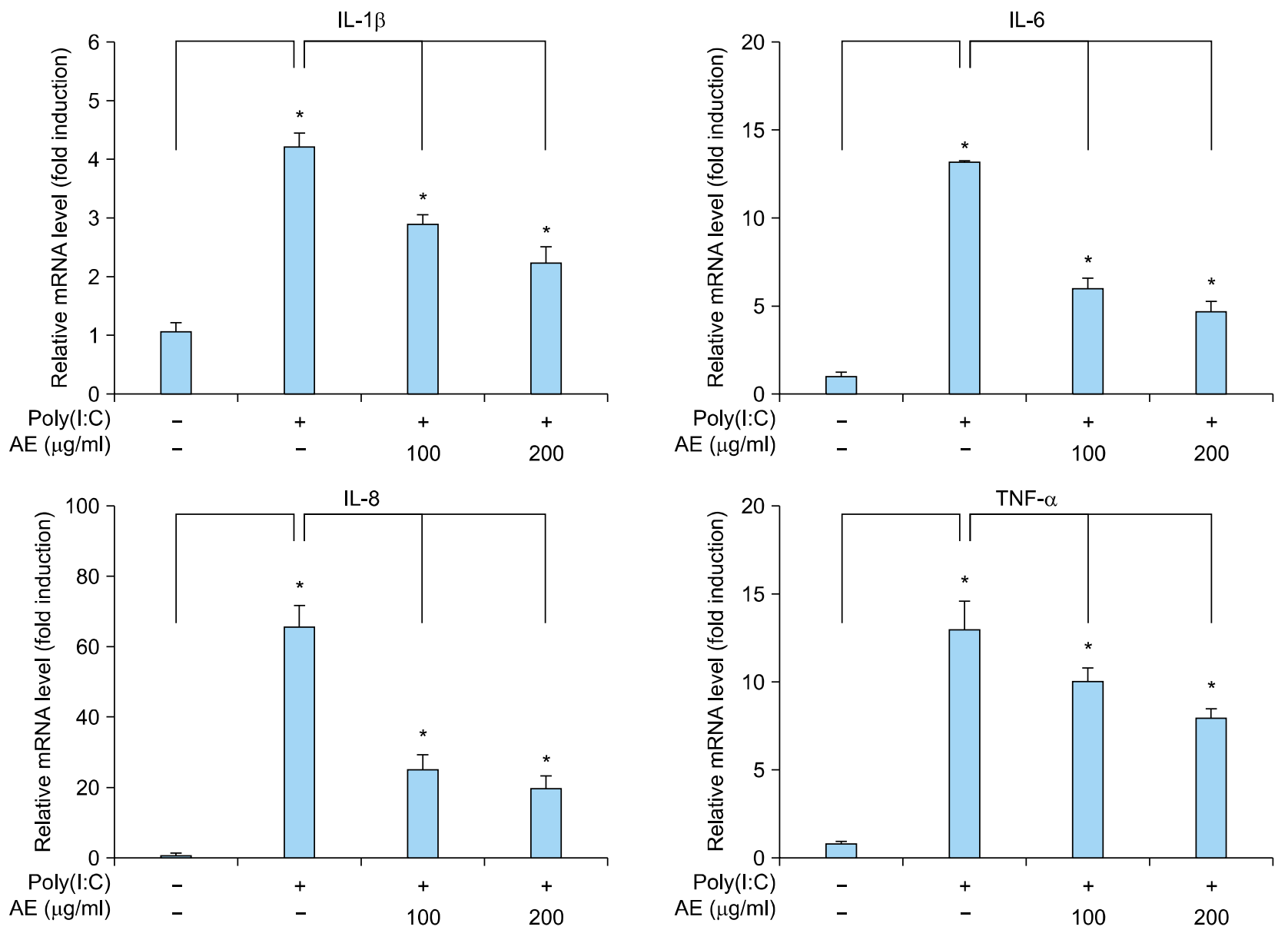

Fig. 2. Effect of Ampelopsis japonica Makino extract (AE) on poly(I:C)-induced inflammatory reaction in keratinocytes. SV40Tag-transformed human epidermal keratinocytes were pretreated with $\mathrm{AE}$ at the indicated concentrations for 1 hour, and then stimulated with $1 \mu \mathrm{g} / \mathrm{ml}$ poly(l:C) for 2 hours. The messenger RNA (mRNA) level was determined by quantitative real-time polymerase chain reaction. Data are expressed as fold induction. The mean values \pm standard deviations are averages of triplicate measurements. IL: interleukin, TNF- $\alpha$ : tumor necrosis factor- $\alpha .{ }^{*} p<0.01$. 

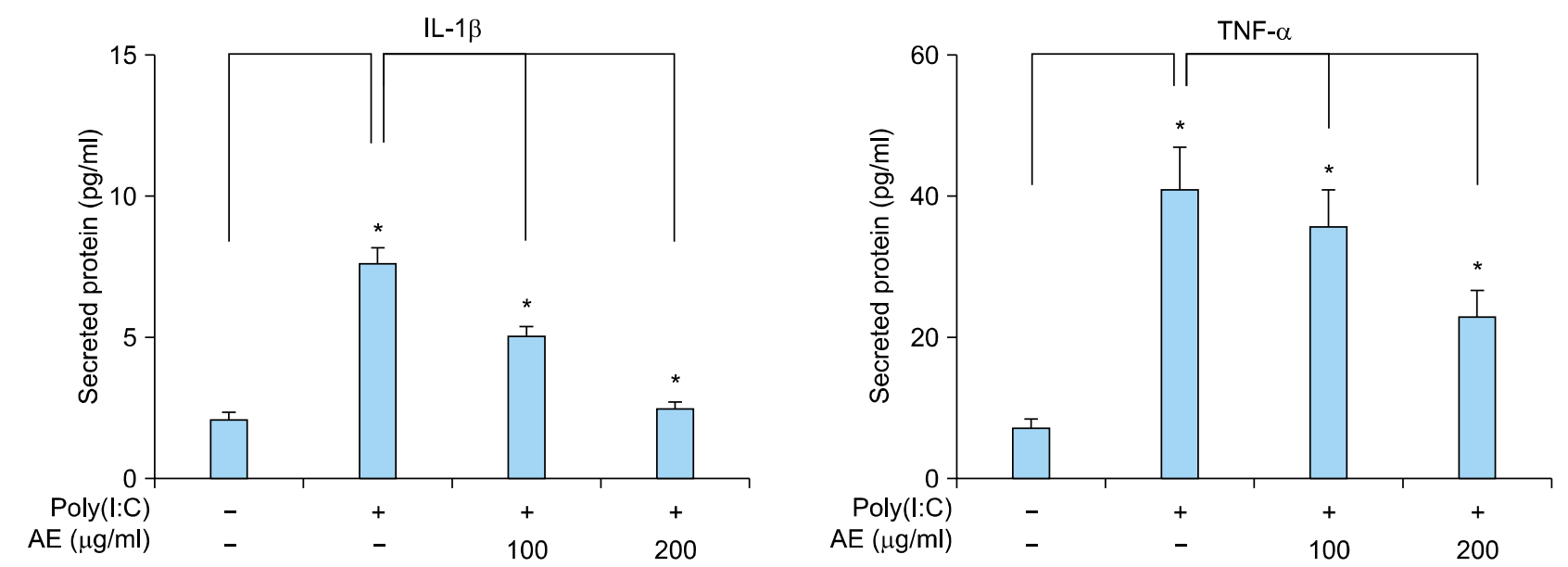

Fig. 3. Effect of Ampelopsis japonica Makino extract (AE) on poly(l:C)-induced cytokine release from keratinocytes. SV40Tag-transformed human epidermal keratinocytes were pretreated with $\mathrm{AE}$ at the indicated concentrations for 1 hour, and then stimulated with $1 \mu \mathrm{g} / \mathrm{ml}$ poly $(\mathrm{l}: \mathrm{C})$ for 24 hours. Released cytokines were measured by enzyme-linked immunosorbent assay. The mean values \pm standard deviations are averages of triplicate measurements. IL: interleukin, TNF- $\alpha$ : tumor necrosis factor- $\alpha .{ }^{*} p<0.01$.

A

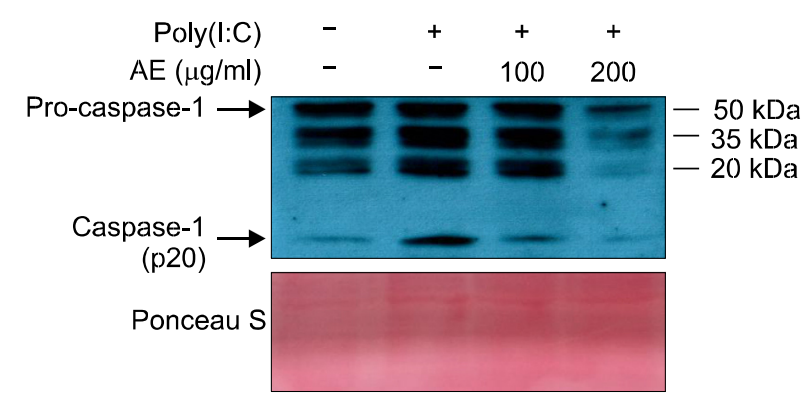

B

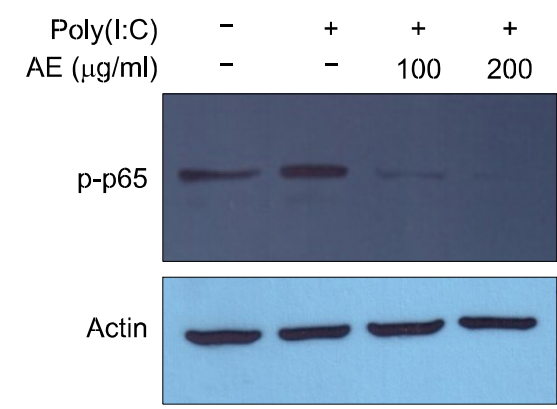

Fig. 4. (A) Effect of Ampelopsis japonica Makino extract (AE) on poly(l:C)-induced inflammasome activation in keratinocytes. SV40Tag-transformed human epidermal keratinocytes (SV-HKEs) were pretreated with AE at the indicated concentrations for 1 hour, and then stimulated with $1 \mu \mathrm{g} / \mathrm{ml}$ poly $(\mathrm{l}: \mathrm{C})$ for 24 hours. Culture medium was collected and concentrated, then subjected to Western blot. The protein level for active form of caspase-1 (p20) was decreased by AE treatment. Ponceau S staining was used for loading control. (B) Effect of $\mathrm{AE}$ on poly(I:C)-induced nuclear factor (NF)- $\kappa \mathrm{B}$ activation in keratinocytes. SV-HKEs were pretreated with $\mathrm{AE}$ at the indicated concentrations for 1 hour, and then stimulated with $1 \mu \mathrm{g} / \mathrm{ml}$ poly $(\mathrm{l}: \mathrm{C})$ for 45 minutes. Activation of NF- $\kappa \mathrm{B}$ signaling was determined by Western blot. The protein level for phoshorylated-p65 (p-p65) NF- $\kappa$ B subunit was decreased by AE treatment. Actin was used for internal control.

clearly showed that AE prevented imiquimod-induced epidermal hyperplasia. Pretreatment of AE resulted in slight reduction of imiquimod-induced IL-1 $\beta$ expression. And, $\mathrm{AE}$ pretreatment also inhibited imiquimod-induced increase of cell proliferation in epidermis, evidenced by reduction of PCNA positive cells (Fig. 5A). Quantification confirmed that imiquimod-induced epidermal thickening was markedly inhibited by AE (Fig. 5B).

\section{DISCUSSION}

Skin is the primary defense organ against environmental insults. Fundamentally, defense mechanism can be pro- vided by the physical and biological methods. Former method is the barrier structure formed by corneocytes and lipid molecules. Later method is the immune system that recognizes foreign materials and eliminates them. Recent concept suggests that two of defense methods can be provided by the epidermal keratinocytes. With regard to the role of immune cells, keratinocytes express many TLR molecules and engage in innate immune response ${ }^{6}$. In condition related to the skin diseases, keratinocytes are susceptible to external stimuli and show excessive response. Well-known example includes psoriasis, in which keratinocytes produce a range of inflammatory cytokines, thereby functioning to recruit and activate immune cells such 
A
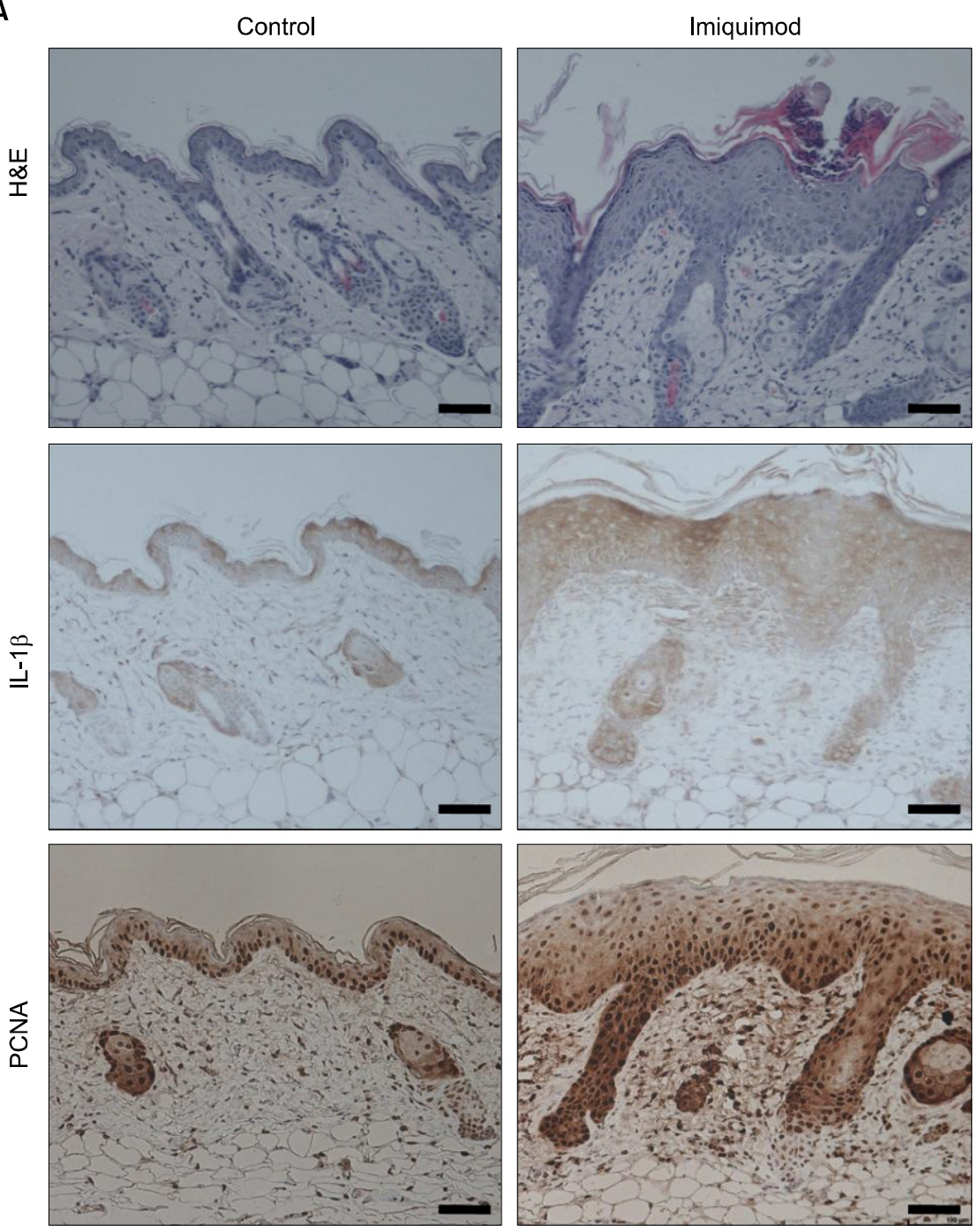
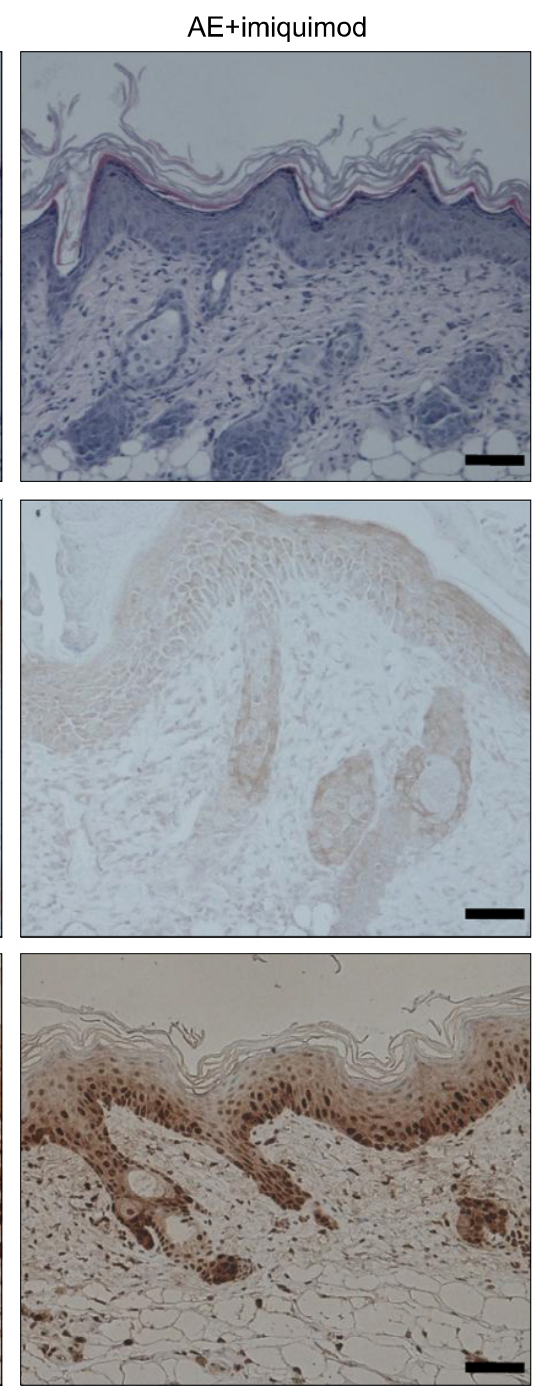

B

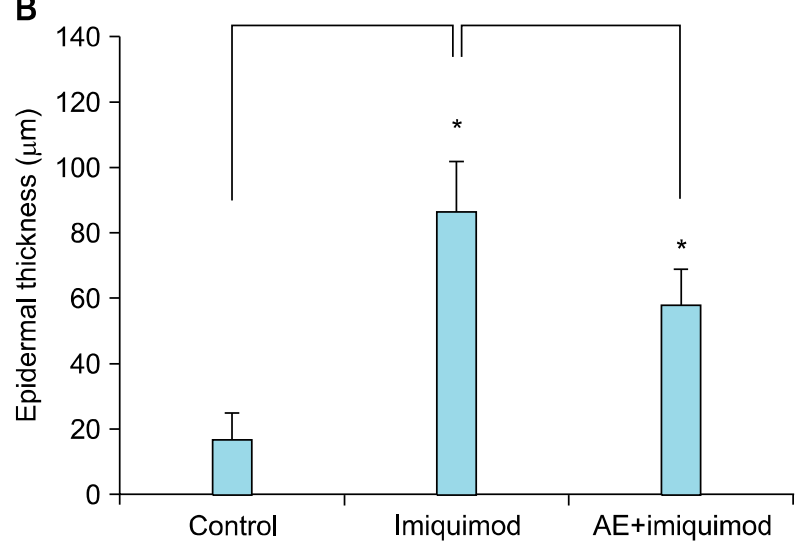

as neutrophils and activated $\mathrm{T}_{\text {cells }}{ }^{18}$. Thus, the approach that inhibits inflammatory reaction in keratinocytes is good method for treatment of psoriasis. In this study, we showed that AE has an anti-inflammatory activity in keratinocytes that challenged with PAMPs such as poly(l:C). We
Fig. 5. Inhibition of imiquimod-induced psoriasiform dermatitis by Ampelopsis japonica Makino extract (AE). (A) BALB/C mice were topically applied with $5 \%$ imiquimod cream (Aldara; $3 \mathrm{M}$ Health Care Ltd., Leicestershire, United Kingdom) for 7 days. $\mathrm{AE}(1 \mathrm{mg} / \mathrm{ml}$ dissolved in polyethylene glycol) was pretreated 1 hour before imiquimod application. Skin specimens were investigated by hematoxylin and eosin staining (upper panels). Immunohistochemistry analysis was performed to determine the interleukin (IL)- $1 \beta$ level (middle panels), and to determine the cell proliferation in epidermis using anti-proliferating cell nuclear antigen (PCNA) antibody (lower panels). Bar $=50 \mu \mathrm{m}$. (B) Epidermal thickness was measured. Data are the mean values \pm standard deviations $(\mathrm{n}=5)$. ${ }^{*} p<0.01$.

also showed that $A E$ reduced imiquimod-induced epidermal hyperplasia using animal model. Our data suggest that $\mathrm{AE}$ can be developed to the therapeutics for psoriasis. Ampelopsis japonica Makino is a medicinal herb that has been widely used in oriental medicine. Its constituents in- 
clude schizandriside, resveratrol, catechins, epicatechin gallate $^{19}$. Multiple pharmacological activities, including anti-metastatic and neuro-protective effect, have been reported. For example, Ampelopsis japonica ethanol extract significantly suppressed the migration and invasion of MDA-MB-231 breast cancer cells in vitro and inhibited the expression of metalloproteinase (MMP)-2 and MMP- $9^{13}$. Other evidence shows that Ampelopsis japonica extract protects dopaminergic neurons against 1-methyl-4-phenylpyridinium/1-methyl-4-phenyl-1,2,3,6-tetrahydropyridine-induced toxicity in Parkinson's disease models in vitro and in vivo ${ }^{20}$.

In this study, we demonstrated that $\mathrm{AE}$ inhibited poly(I:C)-induced inflammatory reaction of cultured keratinocytes, and also that AE pretreatment inhibited imiquimod-induced psoriasiform dermatitis in mouse model. Furthermore, AE significantly inhibited NF- $\kappa$ B activation. In inflammatory reaction, the intracellular signaling molecule NF- $\kappa$ B exerts its pivotal role as a central transcriptional regulator, which directly binds to promoter of many inflammatory cytokine genes. Thus, it can be speculated that anti-inflammatory effect of AE may be due to its action on intracellular signaling cascade. At the moment, it is not clear what components of $\mathrm{AE}$ are the active ingredients to show anti-inflammatory potential. It has been known that Ampelopsis japonica contains many of biologically active ingredients. Of those, epicatechin gallate and catechin gallate have been shown to have superior anti-inflammatory potential in various cell types such as pancreatic tumor cells and dental pulp cells ${ }^{21,22}$. We speculate that these components of AE can also affect the inflammatory reaction of skin keratinocytes induced by PAMPs, because that somehow common intracellular signaling event is occurred in inflammatory reaction irrespective of cell types. Elucidation of active ingredients will be an interesting further study.

In summary, we demonstrated that $\mathrm{AE}$ has a potential for inhibiting PAMPs-induced innate immune response of keratinocytes in vitro and in vivo. Our data suggest that $\mathrm{AE}$ would be a promising candidate for the treatment of psoriasis.

\section{ACKNOWLEDGMENT}

This study was supported by a grant of the Korean Health Technology R\&D Project, Ministry of Health and Welfare, Republic of Korea (HN12C0062).

\section{REFERENCES}

1. Stalder JF, Tennstedt D, Deleuran M, Fabbrocini G, de
Lucas $R$, Haftek $M$, et al. Fragility of epidermis and its consequence in dermatology. J Eur Acad Dermatol Venereol 2014;28(Suppl 4):1-18.

2. Rice $\mathrm{RH}, \mathrm{Green} \mathrm{H}$. The cornified envelope of terminally differentiated human epidermal keratinocytes consists of cross-linked protein. Cell 1977; 11:417-422.

3. Steinert PM, Marekov LN. The proteins elafin, filaggrin, keratin intermediate filaments, loricrin, and small prolinerich proteins 1 and 2 are isodipeptide cross-linked components of the human epidermal cornified cell envelope. J Biol Chem 1995;270:17702-17711.

4. Nemes Z, Steinert PM. Bricks and mortar of the epidermal barrier. Exp Mol Med 1999;31:5-19.

5. Medzhitov R. Toll-like receptors and innate immunity. Nat Rev Immunol 2001;1:135-145.

6. McInturff JE, Modlin RL, Kim J. The role of toll-like receptors in the pathogenesis and treatment of dermatological disease. J Invest Dermatol 2005;125:1-8.

7. Trinchieri G, Sher A. Cooperation of Toll-like receptor signals in innate immune defence. Nat Rev Immunol 2007; 7:179-190.

8. Lowes MA, Bowcock AM, Krueger JG. Pathogenesis and therapy of psoriasis. Nature 2007;445:866-873.

9. Valdimarsson H, Bake BS, Jónsdótdr I, Fry L. Psoriasis: a disease of abnormal Keratinocyte proliferation induced by $T$ lymphocytes. Immunol Today 1986;7:256-259.

10. Lebre MC, van der Aar AM, van Baarsen L, van Capel TM, Schuitemaker JH, Kapsenberg ML, et al. Human keratinocytes express functional Toll-like receptor 3, 4, 5, and 9. J Invest Dermatol 2007;127:331-341.

11. Prens EP, Kant M, van Dijk G, van der Wel LI, Mourits $S$, van der Fits L. IFN-alpha enhances poly-IC responses in human keratinocytes by inducing expression of cytosolic innate RNA receptors: relevance for psoriasis. J Invest Dermatol 2008;128:932-938.

12. Chen X, Takai T, Xie Y, Niyonsaba F, Okumura K, Ogawa H. Human antimicrobial peptide LL-37 modulates proinflammatory responses induced by cytokine milieus and double-stranded RNA in human keratinocytes. Biochem Biophys Res Commun 2013;433:532-537.

13. Nho KJ, Chun JM, Kim DS, Kim HK. Ampelopsis japonica ethanol extract suppresses migration and invasion in human MDA-MB-231 breast cancer cells. Mol Med Rep 2015; 11:3722-3728.

14. Andrés RM, Montesinos MC, Navalón P, Payá M, Terencio MC. NF- $\kappa$ B and STAT3 inhibition as a therapeutic strategy in psoriasis: in vitro and in vivo effects of BTH. J Invest Dermatol 2013;133:2362-2371.

15. Grimstad $\varnothing$, Husebye $H$, Espevik T. TLR3 mediates release of $\mathrm{IL}-1 \beta$ and cell death in keratinocytes in a caspase-4 dependent manner. J Dermatol Sci 2013;72:45-53.

16. van der Fits L, Mourits S, Voerman JS, Kant M, Boon L, Laman JD, et al. Imiquimod-induced psoriasis-like skin inflammation in mice is mediated via the IL-23/IL-17 axis. J Immunol 2009;182:5836-5845.

17. Hemmi H, Kaisho T, Takeuchi O, Sato S, Sanjo H, Hoshino $\mathrm{K}$, et al. Small anti-viral compounds activate immune cells 
via the TLR7 MyD88-dependent signaling pathway. Nat Immunol 2002;3:196-200.

18. Sweeney CM, Tobin AM, Kirby B. Innate immunity in the pathogenesis of psoriasis. Arch Dermatol Res 2011;303: 691-705.

19. Kim IH, Umezawa M, Kawahara N, Goda $\mathrm{Y}$. The constituents of the roots of Ampelopsis japonica. J Nat Med 2007;61:224-225.

20. Park H, Shim JS, Kim HG, Lee H, Oh MS. Ampelopsis radix protects dopaminergic neurons against 1-methyl-4-phenylpyridinium/1-methyl-4-phenyl-1,2,3,6-tetrahydropyridine-In duced toxicity in Parkinson's disease models in vitro and in vivo. Evid Based Complement Alternat Med 2013;2013: 346438.

21. Kürbitz C, Heise D, Redmer T, Goumas F, Arlt A, Lemke J, et al. Epicatechin gallate and catechin gallate are superior to epigallocatechin gallate in growth suppression and antiinflammatory activities in pancreatic tumor cells. Cancer Sci 2011; 102:728-734.

22. Nakanishi T, Mukai K, Yumoto $H$, Hirao K, Hosokawa $Y$, Matsuo T. Anti-inflammatory effect of catechin on cultured human dental pulp cells affected by bacteria-derived factors. Eur J Oral Sci 2010;118:145-150. 